\title{
Sacrospinous hysteropexy versus vaginal hysterectomy with suspension of the uterosacral ligaments in women with uterine prolapse stage 2 or higher: multicentre randomised non-inferiority trial
}

\author{
Renée J Detollenaere, ${ }^{1,2}$ Jan den Boon, ${ }^{1}$ Jelle Stekelenburg, ${ }^{3}$ Joanna IntHout, ${ }^{4}$ Mark E Vierhout, ${ }^{2}$ \\ Kirsten B Kluivers, ${ }^{2}$ Hugo W F van Eijndhoven ${ }^{1}$
}

ABSTRACT
OBJECTIVE
To investigate whether uterus preserving vaginal
sacrospinous hysteropexy is non-inferior to vaginal
hysterectomy with suspension of the uterosacral
ligaments in the surgical treatment of uterine prolapse.
DESIGN
Multicentre randomised controlled non-blinded
non-inferiority trial.

\section{SETTING}

4 non-university teaching hospitals, the Netherlands.

\section{PARTICIPANTS}

208 healthy women with uterine prolapse stage 2 or higher requiring surgery and no history of pelvic floor surgery.

\section{INTERVENTIONS}

Treatment with sacrospinous hysteropexy or vaginal hysterectomy with suspension of the uterosacral ligaments. The predefined non-inferiority margin was an increase in surgical failure rate of $7 \%$.

\section{MAIN OUTCOME MEASURES}

Primary outcome was recurrent prolapse stage 2 or higher of the uterus or vaginal vault (apical compartment) evaluated by the pelvic organ prolapse quantification system in combination with bothersome bulge symptoms or repeat surgery for recurrent apical prolapse at 12 months' follow-up. Secondary outcomes were overall anatomical recurrences, including recurrent anterior compartment (bladder) and/or posterior compartment (bowel) prolapse, functional outcome, complications, hospital stay, postoperative recovery, and sexual functioning.

\section{RESULTS}

Sacrospinous hysteropexy was non-inferior for anatomical recurrence of the apical compartment with bothersome bulge symptoms or repeat surgery $(n=0$, $0 \%$ ) compared with vaginal hysterectomy with suspension of the uterosacral ligaments $(n=4,4.0 \%$, difference $-3.9 \%, 95 \%$ confidence interval for difference $-8.6 \%$ to $0.7 \%$ ). At 12 months, overall anatomical recurrences, functional outcome, quality of life, complications, hospital stay, measures on postoperative recovery, and sexual functioning did not differ between the two groups. Five serious adverse events were reported during hospital stay. None was considered to be related to the type of surgery.

\section{CONCLUSIONS}

Uterus preservation by sacrospinous hysteropexy was non-inferior to vaginal hysterectomy with suspension of the uterosacral ligaments for surgical failure of the apical compartment at 12 months' follow-up.

\section{TRIAL REGISTRATION}

Trialregister.nl NTR1866.

\section{Introduction}

Pelvic organ prolapse is a common health problem. The prevalence of such prolapse is as much as $40 \%$ in women aged more than 45 years, with millions of women affected worldwide, and the incidence is still rising as a result of aging populations and increasing obesity rates. ${ }^{1}$ Pelvic organ prolapse has a negative influence on women's quality of life and is associated with physical, psychological, and sexual problems.

The lifetime risk for prolapse surgery is $11-20 \%$, and worldwide vaginal hysterectomy is the most commonly performed surgical procedure for uterine prolapse. ${ }^{2-6}$ Performing a hysterectomy for uterine prolapse is not an evidence based practice and whether or not the uterus should be removed is debatable. Uterus preserving procedures such as vaginal sacrospinous hysteropexy, in which the uterus is attached to the sacrospinous ligament, are becoming more popular. In a recent study we found a trend towards more uterus preservation in the Netherlands, which is in line with more women opting to retain their uterus in case of an equal outcome with hysterectomy. ${ }^{6-9}$ Uterus preservation is thought to be less invasive, and in prospective non-randomised and retrospective cohort studies sacrospinous hysteropexy was as effective as vaginal 
hysterectomy, with a similar rate of recurrence and repeat surgery but with a shorter operating time, less blood loss, faster recovery, and fewer complications. ${ }^{10-12}$ A hysterectomy has known benefits as well: it prevents the development of uterine cancer and stops menstrual bleeding in premenopausal women. After vaginal hysterectomy, however, women may be at increased risk of recurrent prolapse since hysterectomy disrupts the supportive structures of the pelvic floor. ${ }^{13}$ To prevent future prolapse of the vaginal vault after hysterectomy additional vault suspension is recommended. ${ }^{1415}$ Randomised controlled trials comparing uterus preserving techniques and hysterectomy with vault suspension are limited. One randomised controlled trial found more recurrent uterine prolapse with sacrospinous hysteropexy after 12 months, but the recurrence rate was only a secondary endpoint. ${ }^{16}$ Another randomised controlled trial found no differences in sexual functioning after six months but did not report on anatomical outcomes. ${ }^{17}$

Owing to a lack of well executed randomised controlled trials and clear guidelines on the treatment of uterine prolapse, the variation in surgical management of uterine prolapse is enormous and a Cochrane meta-analysis on surgery for pelvic organ prolapse concluded that more research on this subject is needed. ${ }^{18}$ We tested the hypothesis that sacrospinous hysteropexy is non-inferior to vaginal hysterectomy with suspension of the uterosacral ligaments for surgical failure after 12 months' follow-up.

\section{Methods}

\section{Study design}

A detailed version of the trial protocol has been published previously. ${ }^{19}$ In short, all women with uterine prolapse at stage 2 or higher (uterine prolapse $1 \mathrm{~cm}$ above the hymen or beyond) requiring surgery were invited to participate. Participants were randomly assigned to sacrospinous hysteropexy or vaginal hysterectomy with suspension of the uterosacral ligaments in a non-blinded multicentre randomised controlled non-inferiority trial.

Women with coexisting prolapse of the anterior or posterior vaginal wall, or both were able to participate, and cervical elongation together with uterine prolapse was no reason for exclusion. Concomitant repair of anterior or posterior vaginal prolapse (colporrhaphy) was allowed, including anti-incontinence surgery. We excluded women with previous pelvic floor or prolapse surgery, known malignancy or an abnormal cervical smear test result, a wish to preserve fertility, language barriers, immunological or haematological disorders interfering with recovery after surgery, abnormal ultrasound findings of the uterus or ovaries, or abnormal uterine bleeding, and those who were unwilling to return for follow-up.

All four participating centres were Dutch large non-university teaching hospitals. Centres had to offer both treatment modalities, and we invited them to participate if they were known to perform the interventions in the same standardised manner. All gynaecologists were experienced and had performed a minimum of 20 procedures of each intervention before the start of the trial. As the participating hospitals were teaching hospitals, residents were allowed to perform procedures under direct supervision of the gynaecologist. Gynaecologists and residents of the participating centres assessed the eligibility of patients. The decision to treat uterine prolapse surgically was a shared decision by the woman and her gynaecologist.

We gave the women written information and obtained informed consent before randomisation. The women were randomly allocated in a 1:1 ratio using a web based application with computer generated randomisation tables in blocks of four, stratified by hospital and stage of uterine prolapse. The trial was non-blinded as it was impossible to blind surgeons and women to the allocated surgical procedure. An independent doctor who was not involved in treatment carried out the 12 month follow-up.

\section{Outcome measures}

Initially the primary outcome was surgical failure at the 12 month follow-up. As outcome definitions to evaluate prolapse surgery were improved after the start of this trial, we changed the primary outcome during enrolment and before data analysis into the composite outcome measure of recurrent pelvic organ prolapse stage 2 or higher in the apical compartment (uterus or vaginal vault) with bothersome bulge symptoms or repeat surgery for recurrent apical prolapse at the 12 month follow-up. Isala hospital's medical ethical committee approved this change, and during enrolment we published the protocol in an open access online journal. ${ }^{19}$

We evaluated the original primary outcome, overall anatomical failure (pelvic organ prolapse stage 2 or higher in any compartment), as a secondary outcome together with two additional definitions of surgical failure or success. Firstly, a composite outcome of success, defined as no prolapse beyond the hymen, no bothersome bulge symptoms, and no repeat surgery or pessary use for recurrent prolapse within 12 months. Secondly, overall surgical failure: pelvic organ prolapse stage 2 or higher, pessary use, or repeat surgery for recurrent prolapse in any compartment within 12 months. ${ }^{20}$ Other secondary outcomes were functional outcome, quality of life, complications, hospital stay, postoperative recovery, and sexual functioning.

\section{Interventions}

We referred to a detailed description of the study interventions to ensure a uniform technique among the surgeons. ${ }^{19}$ All women received perioperative antibiotics, prophylaxis against thrombosis, and a bladder catheter according to local hospital protocol. Analgesics were given postoperatively in accordance with each hospital's protocol. The women were advised to abstain from heavy physical work for six weeks.

Sacrospinous hysteropexy-Vaginal sacrospinous hysteropexy was performed unilaterally to the right sacrospinous ligament. The posterior vaginal wall was 
incised and the sacrospinous ligament accessed through the pararectal space. Two permanent sutures (Prolene 1.0; Ethicon, Somerville, NJ) were placed under direct vision through the sacrospinous ligament at least $2 \mathrm{~cm}$ from the ischial spine. Additional anterior or posterior vaginal wall repair or incontinence surgery was performed if indicated. The permanent sutures were also placed through the posterior side of the cervix and tightened and the uterus redressed. The posterior vaginal wall was closed with absorbable sutures (Vicryl 2; Ethicon, Somerville, NJ). (Also see www.youtube.com/ watch?v=ySSfy2A1_RM and www.youtube.com/ watch?v=wjct1r37sTw).

Vaginal hysterectomy-The vaginal wall around the cervix was circumcised. After bladder and bowel dissection the anterior and posterior peritoneum were opened. The uterosacral ligaments-strong supportive ligaments that attach the cervix to the sacrumwere identified, ligated, and transected. The uterus was released in several steps using clamps and sutures. After removal of the uterus, the surgical pedicles were inspected for haemostasis and the adnexa inspected. The peritoneum was closed using a delayed absorbable suture (Vicryl 1.0; Ethicon, Somerville, $\mathrm{NJ})$. Additional vault suspension in this study was performed by suspension of the uterosacral ligaments. Such suspension involves the attachment of the uterosacral ligaments to the vaginal vault, thereby restoring normal support to the apical compartment. ${ }^{21}$ Concomitant anterior or posterior vaginal wall repair and anti-incontinence surgery were performed afterwards if indicated.

\section{Measurements and procedures}

Gynaecological examination before surgery included pelvic ultrasonography to exclude uterine or ovarian disease, a cervical smear test, and vaginal inspection in $45^{\circ}$ semi-upright position for staging pelvic organ prolapse using the pelvic organ prolapse quantification system (POP-Q). ${ }^{22}$ This system involves quantitative measurements of various points of the vaginal wall, using the hymen as a reference point. The degree of prolapse of the anterior vaginal wall, posterior vaginal wall, and uterus or vaginal vault is measured in centimetres either above or proximal to the hymen (negative number) or beyond, or distal to the hymen (positive number), with the plane of the hymen being defined as zero. The genital hiatus, perineal body, and total vaginal length are also measured. Based on these measurements, a stage (0-4) is determined for each compartment. The overall stage is equal to the stage of the most severely prolapsed compartment. The women came to the hospital to be examined for pelvic organ prolapse stage at baseline and at six weeks, six months, and 12 months after surgery and annually thereafter until 60 months' follow-up. At the time of the follow-up visits women completed validated health related and disease specific quality of life questionnaires: short form-36, Euroqol 5D, urogenital distress inventory, defecatory distress inventory, and incontinence impact questionnaire. ${ }^{23-26}$ We defined the presence of bothersome bulge symptoms after surgery as a positive answer to any of the following two questions from the urogenital distress inventory: "Do you experience a sensation of bulging or protrusion from the vagina?" and "Do you have a bulge or something falling out that you can see in the vagina?" in combination with a response "somewhat bothered" to "very much bothered" to the question "how much does this bother you?" To assess sexual functioning, we used the 12 item pelvic organ prolapse/urinary incontinence sexual questionnaire, translated from the validated questionnaire but not validated for Dutch language. ${ }^{27}$

During hospital admission and the first six weeks after surgery, the women kept a diary to evaluate postoperative pain (range 0-10), measured by a validated visual analogue scale; analgesics; and postoperative recovery, measured with the validated recovery index-10. ${ }^{28}$

Data were entered and registered using a web based application facilitated by the Dutch consortium for studies in women's health and reproductivity (www. studies-obsgyn.nl).

\section{Statistical analysis}

The sample size for this trial was based on the primary outcome. Recurrence rates in the apical compartment after vaginal hysterectomy vary from $0-12 \%,{ }^{29}$ so that a failure rate including bothersome symptoms and repeat surgery after sacrospinous hysteropexy of $10 \%$ or more might be regarded as high. As we expected a failure rate of $3 \%$ based on outcomes of vaginal hysterectomy in a previous randomised study, ${ }^{1630}$ we set the non-inferiority margin at $7 \%$. This means that when the upper limit of the $95 \%$ confidence interval for the estimated difference in recurrence rate after sacrospinous hysteropexy versus vaginal hysterectomy exceeds $7 \%$, sacrospinous hysteropexy is inferior to vaginal hysterectomy. Assuming an absolute recurrence rate of $3 \%$ in both groups and a two sided $\alpha$ risk of 0.05 , with two groups of 94 women each the trial had $80 \%$ power with a prespecified non-inferiority margin of $7 \%$ to assess non-inferiority of sacrospinous hysteropexy. Considering a $10 \%$ loss to follow-up, we required a 208 women-104 in each study arm.

We assessed study outcomes by intention to treat analysis. In case of missing data on anatomical outcome at 12 months we applied two strategies. For the first by intention to treat analysis, we used the last observation carried forward with data at the six month follow-up visit if available. If these data were not available, we left the women out of the intention to treat-last observation carried forward analysis. For the second by intention to treat analysis we applied conservative imputation for all women with missing data at 12 months (worst case scenario, failure). In the case of missing questionnaires, we obtained information on the presence or absence of bothersome bulge symptoms from the 12 month follow-up visit.

We also carried out a per protocol analysis on the primary and secondary outcomes for anatomical and 


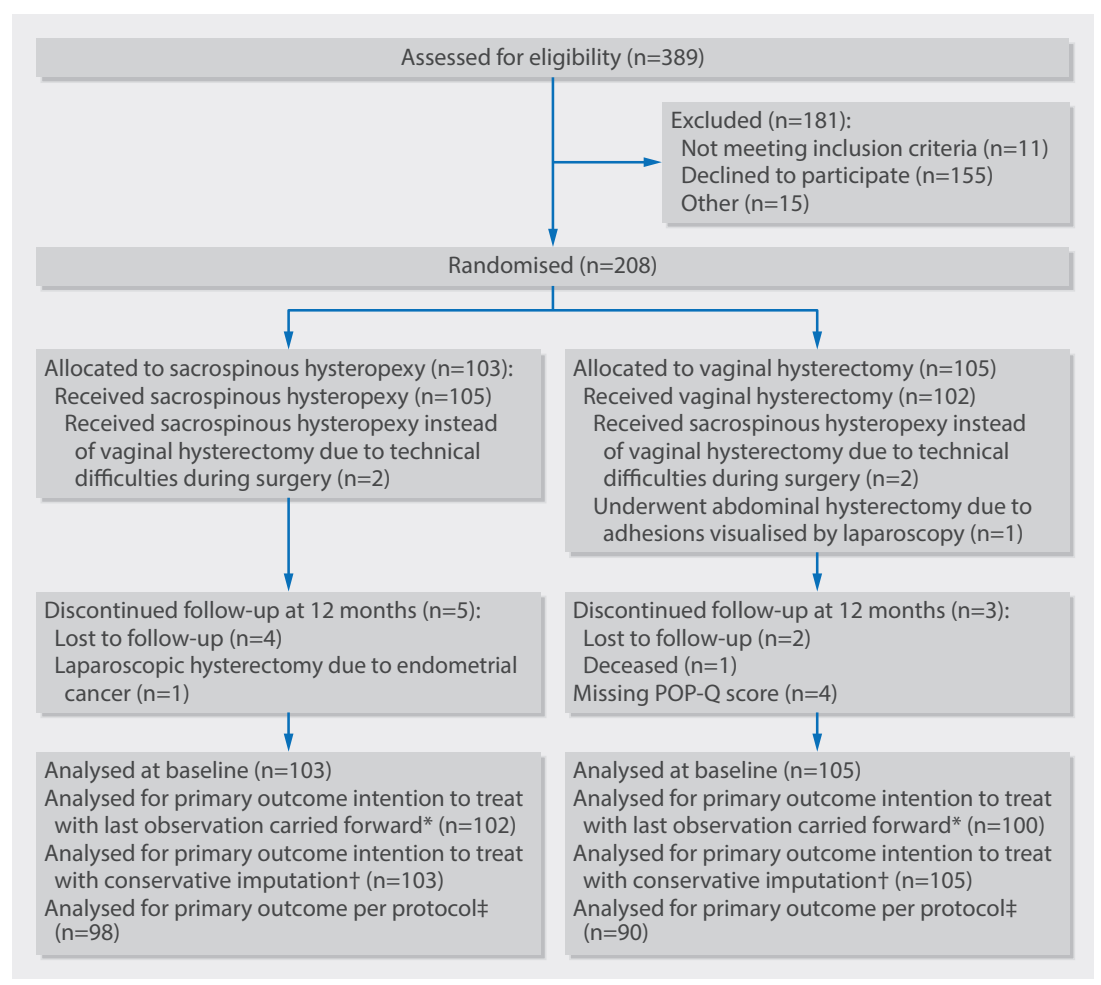

Flow of women through study. *Intention to treat: two patients allocated to vaginal hysterectomy $(\mathrm{VH})$ received sacrospinous hysteropexy $(\mathrm{SH})$ and were analysed in the $\mathrm{VH}$ group. Data at six and 12 month follow up were missing in one patient after $\mathrm{SH}$ and six patients after $\mathrm{VH}$; one patient after $\mathrm{VH}$ had recurrent apical prolapse but pelvic organ prolapse quantification (POP-Q) score was missing, this patient was included in the intention to treat-last observation carried forward analysis. + Missed data imputed as failure. $¥$ Per protocol analysis: two patients did not receive intended treatment. Excluded per protocol analysis: lost for follow-up at 12 months $(n=8)$, missing or incomplete POP-Q score $(n=5)$, and major protocol deviations $(n=9)$; two patients met two criteria to be excluded from per protocol analysis hysteropexy ( $\mathrm{n}=103)$ or to vaginal hysterectomy $(\mathrm{n}=105)$. The figure shows the flow of women through the study. Baseline characteristics were similar between the groups (table 1) and pelvic measurements and characteristics did not differ at baseline (table 2).

Table 3 presents the results on the primary outcome and the additional definitions of surgical failure. Sacrospinous hysteropexy was non-inferior to vaginal hysterectomy for anatomical recurrence of the apical compartment with bothersome bulge symptoms or repeat surgery for recurrent apical prolapse: sacrospinous hysteropexy $0 \%(n=0)$ versus vaginal hysterectomy $4.0 \%(n=4)$, difference $-3.9 \%$ (95\% confidence interval $-8.6 \%$ to $0.7 \%$ ) for the intention to treat-last observation carried forward approach. Non-inferiority of sacrospinous hysteropexy was also shown in the intention to treat analysis with conservative imputation and the per protocol analysis. The original primary outcome variable of overall anatomical failure occurred in $50 \%$ of the women after sacrospinous hysteropexy compared with $44 \%$ after vaginal hysterectomy (95\% confidence interval for difference $-7.4 \%$ to $20.1 \%$ ). No notable differences were found for anatomical recurrences in the different compartments, except for the posterior vaginal wall: sacrospinous hysteropexy 4\% versus vaginal hysterectomy 14\% (95\% confidence interval for difference $-18.2 \%$ to $-1.8 \%$ ).

Table 4 shows the intraoperative and postoperative details of the women, including the secondary outcomes of complication rate and length of hospital stay. Five serious adverse events were reported during hospital stay: two after vaginal hysterectomy and three after sacrospinous hysteropexy. One woman developed paralytic ileus after vaginal hysterectomy. She had also experienced this problem after orthopaedic surgery. She aspirated gastric contents eight days after surgery, developed aspiration pneumonia, and died because of multi-organ failure. The other serious adverse events were atrial fibrillation, which required cardioversion (vaginal hysterectomy); stroke two days after surgery, but with full recovery and no loss of function (sacrospinous hysteropexy); postoperative pneumonia (sacrospinous hysteropexy); and anaphylactic reaction to prophylactic antibiotics before the surgical procedure (sacrospinous hysteropexy); in this last woman the surgical procedure was postponed for several months, without any problems. None of the serious adverse events were judged to be related to the type of surgery.

Tables 5 and 6 provide details on the other secondary outcomes. Functional outcome and quality of life did not differ significantly between the groups (table 5). Postoperative recovery was similar after both interventions, with comparable recovery index-10 scores at 1, 2, 4 , and 6 weeks after surgery (table 6). Among the patients who completed the pelvic organ prolapse/urinary incontinence sexual questionnaire before and after surgery, there was significant improvement in scores in both surgical groups $(\mathrm{P}<0.002$ each) but no significant difference in total scores between both interventions (table 6). 


\begin{tabular}{|c|c|c|}
\hline Characteristics & $\begin{array}{l}\text { Sacrospinous } \\
\text { hysteropexy }(n=103)\end{array}$ & $\begin{array}{l}\text { Vaginal hysterectomy } \\
(n=105)\end{array}$ \\
\hline Median (range) age (years) & $62.7(45-85)$ & $61.9(33-82)$ \\
\hline \multicolumn{3}{|l|}{ Highest educational level: } \\
\hline Primary or secondary school & $14(14)$ & $7(7)$ \\
\hline High school & $78(77)$ & $82(80)$ \\
\hline Bachelor, master, or academic degree & $9(9)$ & $13(13)$ \\
\hline \multicolumn{3}{|l|}{ Comorbidity: } \\
\hline Cardiovascular disease & $39(38)$ & $32(31)$ \\
\hline Diabetes mellitus & $5(5)$ & $5(5)$ \\
\hline Respiratory disease & $3(3)$ & $7(7)$ \\
\hline Current smoker & $13(14)$ & $9(10)$ \\
\hline Median (range) No of vaginal deliveries & $2(0-7)$ & $3(0-7)$ \\
\hline Median (range) No of caesarean deliveries & $0(0-1)$ & $0(0-2)$ \\
\hline Mean (SD) body mass index & $26.0(3.3)$ & $25.9(3.5)$ \\
\hline
\end{tabular}

Pain scores on the visual analogue scale did not differ notably between both interventions, except for day 14 in favour of hysterectomy. In eight out of nine women who experienced buttock pain, a typically reported problem after sacrospinous hysteropexy, the pain resolved (visual analogue scale score $<2$ ) spontaneously within the first six weeks. One woman underwent suture cutting and vaginal hysterectomy after four months because of persistent pain localised at the

\begin{tabular}{|c|c|c|}
\hline Characteristics & $\begin{array}{l}\text { Sacrospinous } \\
\text { hysteropexy }(n=103)\end{array}$ & $\begin{array}{l}\text { Vaginal hysterectomy } \\
(\mathrm{n}=105)\end{array}$ \\
\hline \multicolumn{3}{|c|}{ POP-Q stage uterine prolapse (point C)*: } \\
\hline 2 & $67(65)$ & $66(63)$ \\
\hline 3 & $28(28)$ & $29(28)$ \\
\hline 4 & $8(8)$ & $10(10)$ \\
\hline \multicolumn{3}{|l|}{ POP-Q stage 2-4: } \\
\hline Anterior prolapse $(\mathrm{Ba} \geq-1)$ & $94(94)$ & $95(92)$ \\
\hline Posterior prolapse $(\mathrm{Bp} \geq-1)$ & $29(29)$ & $33(32)$ \\
\hline \multicolumn{3}{|l|}{ Prolapse beyond hymen: } \\
\hline Apical (POP-Q C >0) & $48(48)$ & $43(42)$ \\
\hline Anterior (POP-Q Aa or $\mathrm{Ba}>0$ ) & $71(71)$ & $72(70)$ \\
\hline Posterior (POP-Q Ap or Bp >0) & $11(11)$ & $11(11)$ \\
\hline Overall POP-Q stage*: & $\mathrm{n}=100$ & $n=103$ \\
\hline 2 & $25(25)$ & $36(35)$ \\
\hline 3 & $70(70)$ & $62(60)$ \\
\hline 4 & $5(5)$ & $5(5)$ \\
\hline \multicolumn{3}{|l|}{ Vaginal bulge symptoms: } \\
\hline Any & 94/101 (93) & $98 / 103(95)$ \\
\hline Bothersome & 93/100 (93)† & 96/101 (95) \\
\hline
\end{tabular}

POP-Q=pelvic organ prolapse quantification.

Percentages were calculated using non-missing data. All patients were analysed as allocated. Five POP-Q scores were missing at baseline.

*System involves quantitative measurements of various points of vaginal wall with hymen as reference point. Degree of prolapse of anterior vaginal wall ( $\mathrm{Aa}$ and $\mathrm{Ba}$ ), posterior vaginal wall ( $\mathrm{Ap}$ and $\mathrm{Bp}$ ), and uterus or vaginal vault (C) measured in centimetres both above or proximal to hymen (negative number) or beyond or distal to hymen (positive number), with plane of hymen defined as zero. A represents the descent of a measurement point $3 \mathrm{~cm}$ proximal to the hymen on the anterior $(\mathrm{Aa})$ and posterior ( $\mathrm{Ap}$ ) vaginal wall. $\mathrm{B}$ is the most descended edge on the anterior $(\mathrm{Ba})$ and posterior $(\mathrm{BP})$ vaginal wall. $\mathrm{POP}-\mathrm{Q}$ stage 2 : most distal prolapse is between $1 \mathrm{~cm}$ above and $1 \mathrm{~cm}$ beyond hymen; stage 3: most distal prolapse is prolapsed $>1 \mathrm{~cm}$ beyond hymen but no further than $2 \mathrm{~cm}$ less than total vaginal length; stage 4: total prolapse.

tNot all women reported bothersome symptoms at baseline. Questionnaire was provided after women

consented to participate, therefore amount of bother as reported at outpatient clinic could differ. place of the sacrospinous hysteropexy sutures. After this procedure she was free of symptoms.

Fourteen protocol deviations occurred: two women received sacrospinous hysteropexy instead of vaginal hysterectomy owing to technical difficulties during surgery (crossovers). In one woman allocated to vaginal hysterectomy, laparoscopic cystectomy before vaginal hysterectomy showed intra-abdominal adhesions, and an abdominal hysterectomy was performed. In two women an exclusion criterion was ignored before randomisation: one woman had had previous pelvic floor surgery (repair of posterior vaginal wall prolapse) and another had primary sclerosing cholangitis with thrombocytopenia. Three women who were assigned to sacrospinous hysteropexy had abnormal smear test results and were treated with electrosurgical excision $(n=2)$ during prolapse surgery or follow-up. In six of 102 women (6\%) apical suspension after vaginal hysterectomy was performed using a McCall procedure instead of suspension of the uterosacral ligaments. The other 96 women underwent suspension of the uterosacral ligaments (94\%). According to the intention to treat principle, we included these women in the intention to treat analysis, with all women analysed as randomised. In the per protocol analysis we excluded these women, except for those classed as crossovers $(n=2)$, as the primary outcome was related to efficacy and these women had no other protocol deviations, and those with abnormal smear test results $(n=3)$, as this was regarded as a minor protocol deviation.

Residents performed three of the procedures in the sacrospinous hysteropexy group (3\%) and 19 in the vaginal hysterectomy group (18\%). Overall surgical failure rate (recurrent prolapse, pessary use, or repeat surgery) did not differ significantly by gynaecologist or resident (91 failures out of 180 procedures (50.6\%) versus nine failures out of 19 procedures (47.4\%), respectively, $\mathrm{P}=0.81$ ). Endometrial cancer was diagnosed during follow-up in one woman in the sacrospinous hysteropexy group (1.0\%), and a laparoscopic hysterectomy was performed.

Urinary retention, defined as more than $150 \mathrm{~mL}$ urine retention after removal of the catheter, was similar between the groups (table 4). Affected women received a transurethral catheter or were instructed to perform clean intermittent self catheterisation. In all women spontaneous micturition was achieved after a maximum length of catheterisation of 40 days (median 5.0 days, range 1-40 days). Subsequent surgical treatment for stress urinary incontinence after 12 months was carried out in 1 of 102 women (1.0\%) after sacrospinous hysteropexy and 4 of 102 women (3.9\%) after vaginal hysterectomy $(\mathrm{P}=0.37)$.

\section{Discussion}

Treatment with sacrospinous hysteropexy was non-inferior to vaginal hysterectomy with suspension of the uterosacral ligaments for surgical failure of the apical compartment in both an intention to treat analysis and a per protocol analysis. We found no notable differences 


\begin{tabular}{|c|c|c|c|}
\hline Outcomes & $\begin{array}{l}\text { Sacrospinous } \\
\text { hysteropexy }\end{array}$ & $\begin{array}{l}\text { Vaginal } \\
\text { hysterectomy }\end{array}$ & Difference $(95 \% \mathrm{Cl})$ \\
\hline \multicolumn{4}{|l|}{ Primary outcome* } \\
\hline ITT analysis with LOCF & 0/102 (0) & $4 / 100(4)$ & $-3.9(-8.6$ to 0.7$)$ \\
\hline $\begin{array}{l}\text { ITT analysis with conservative } \\
\text { imputation }\end{array}$ & $6 / 103(6)$ & 10/105 (10) & $-3.6(-11.2$ to 3.9$)$ \\
\hline Per protocol analysis & $0 / 98(0)$ & $3 / 90(3)$ & $-3.3(-8.0$ to 1.3$)$ \\
\hline \multicolumn{4}{|l|}{ Overall surgical failuret } \\
\hline ITT analysis with LOCF & $52 / 102(51)$ & 49/100 (49) & $1.9(-11.8$ to 15.7$)$ \\
\hline $\begin{array}{l}\text { ITT analysis with conservative } \\
\text { imputation }\end{array}$ & $55 / 103(53)$ & $54 / 105(51)$ & $1.9(-11.6$ to 15.5$)$ \\
\hline Per protocol analysis & $51 / 98(52)$ & 44/90 (49) & $3.1(-11.2$ to 17.4$)$ \\
\hline \multicolumn{4}{|l|}{ Composite outcome successł } \\
\hline ITT analysis with LOCF & $91 / 102(89)$ & $83 / 100(83)$ & $6.1(-3.6$ to 15.8$)$ \\
\hline $\begin{array}{l}\text { ITT analysis with conservative } \\
\text { imputation }\end{array}$ & 87/103 (84) & $82 / 105(78)$ & $6.2(-4.5$ to 16.9$)$ \\
\hline Per protocol analysis & 87/98 (89) & $75 / 90(83)$ & $5.3(-4.7$ to 15.5$)$ \\
\hline \multicolumn{4}{|l|}{ Anatomical failure§ } \\
\hline Overall anatomical failure: & $51 / 101(50)$ & $44 / 100(44)$ & $6.4(-7.4$ to 20.1$)$ \\
\hline Apical compartment & $2 / 102(2)$ & $7 / 100(7)$ & $-5.0(-11.1$ to 1.2$)$ \\
\hline Anterior compartment & 47/101 (47) & 33/99 (33) & $12.9(-0.5$ to 26.4$)$ \\
\hline Posterior compartment & 4/101 (4) & $14 / 99(14)$ & $-10.0(-18.2$ to -1.8$)$ \\
\hline \multicolumn{4}{|l|}{ Prolapse beyond hymenף } \\
\hline Apical (POP-Q C >0) & 0/102 (0) & $4 / 100(4)$ & $-3.9(-8.6$ to 0.7$)$ \\
\hline Anterior (POP-Q Ba >0) & $8 / 101(8)$ & $6 / 99(6)$ & $1.8(-5.6$ to 9.2$)$ \\
\hline Posterior (POP-Q Bp >0) & $0 / 101(0)$ & $2 / 99(2)$ & $-2.0(-5.9$ to 1.9$)$ \\
\hline \multicolumn{4}{|l|}{ Repeat surgeryף } \\
\hline Recurrent prolapse & 1/102 (1) & 4/102 (4) & $-2.9(-7.8$ to 2.0$)$ \\
\hline Apical compartment & $0 / 102(0)$ & $2 / 102(2)$ & $-1.9(-5.7$ to 1.8$)$ \\
\hline Anterior compartment & $1 / 102(1)$ & $4 / 102(4)$ & $-2.9(-7.8$ to 2.0$)$ \\
\hline Posterior compartment & $0 / 102(0)$ & $1 / 102(1)$ & $-1.0(-4.2$ to 2.3$)$ \\
\hline Primary surgery different site $e^{\star \star}$ & $0 / 102(0)$ & $3 / 102(3)$ & $-2.9(-7.1$ to 1.3$)$ \\
\hline \multicolumn{4}{|c|}{ Surgery for non-prolapse conditions } \\
\hline Anti-incontinence & $1 / 102(1)$ & $4 / 102(4)$ & $-2.9(-7.8$ to 2.0$)$ \\
\hline Hysterectomy & $2 / 100(2)$ & - & - \\
\hline \multicolumn{4}{|c|}{$\begin{array}{l}\text { ITT=intention to treat; } L O C F=\text { last observation carried forward; POP-Q=pelvic organ prolapse quantification. } \\
\text { Percentages were calculated using non-missing data. Agresti-Coull method used to calculate } 95 \% \text { confidence } \\
\text { intervals.31 } \\
\text { *Recurrent apical prolapse stage } \geq 2 \text { with bothersome symptoms or repeat surgery for apical prolapse. } \\
\text { tProlapse POP-Q stage } \geq 2 \text { (any compartment) or repeat surgery or pessary use. } \\
\text { fNo prolapse beyond hymen, absence of bothersome bulge symptoms, and no repeat surgery or pessary use. } \\
\text { \$Prolapse POP-Q stage } \geq 2 \text {. } \\
\text { १IIT with LOCF. } \\
\text { **Reoperation for pelvic organ prolapse in non-operated compartment. }\end{array}$} \\
\hline
\end{tabular}

in overall anatomical and surgical failure, functional outcome, quality of life, complications, postoperative recovery, length of hospital stay, and sexual functioning between the interventions. Women who underwent sacrospinous hysteropexy reported more buttock pain after surgery, but pain scores on the visual analogue scale were low and in most cases the pain resolved within six weeks.

\section{Strengths and limitations of this study}

A major strength of this study is its randomised multicentre design and sufficient study population. This is to our best knowledge the first randomised trial to compare uterus preservation with hysterectomy on a large scale using clinically relevant outcome measures.

The study also has some limitations. Firstly, our findings are based on a relatively short follow-up period of 12 months. However, results from registry studies suggest that the highest risk of prolapse surgery after hysterectomy is in the first two postoperative years. Furthermore, not only is long term surgical outcome important but also short term secondary outcomes, such as complication rate and postoperative recovery. ${ }^{33} 34$ The women consented to follow-up for 60 months after surgery, and these data will be further analysed.

After vaginal hysterectomy the ligatures of the uterosacral ligaments were to be sutured to the vaginal vault to aid in long term vaginal support. However, the protocol was ignored in six women and the McCall procedure was performed instead of suspension of the uterosacral ligaments. ${ }^{35}$ Both procedures rely on the uterosacral ligaments for support of the vaginal apex but are considered different treatment modalities for suspension of the vaginal vault, which could have led to differences between treatments. As far as we know, strong evidence on the best technique for vault suspension after vaginal hysterectomy is lacking in the literature, and a recent published trial found similar outcomes after suspension of the uterosacral ligaments and sacrospinous fixation for apical prolapse. ${ }^{36}$ In the per protocol analysis these women were excluded, but this did not alter the conclusions.

Another limitation might be that residents were allowed to perform sacrospinous hysteropexy or vaginal hysterectomy under direct supervision of a gynaecologist because of their training position. Surgery by residents may have led to variation in procedures. In the hysterectomy group more procedures were performed by residents. No statistically significant difference was found in surgical failure rate in women who underwent surgery by either gynaecologist or resident, but the higher number of procedures performed by residents could have contributed to a longer operation time in the vaginal hysterectomy group. Surgery performed by residents may improve the generalisability of the trial findings as their involvement in treatment of pelvic organ prolapse is common in Dutch urogynaecological practice.

\section{Comparison with other studies}

The anatomical outcome after sacrospinous hysteropexy in our study is in line with that of previous studies, ${ }^{1637}$ although the only previous randomised study showed opposite results: Dietz and colleagues found a higher rate of anatomical recurrence of the apical compartment after sacrospinous hysteropexy (7 of 34 (21\%) versus 1 in 31 (3\%) after vaginal hysterectomy, $\mathrm{P}=0.03$ ) after 12 months' follow-up. Possible explanations for this difference might relate to a different surgical protocol or skills, precise definition of the recurrence outcome, and sample size. The primary outcome in the study reported by Dietz and colleagues was recovery time instead of anatomical outcome. These authors considered a pelvic organ prolapse stage 2 or higher of the uterus or vaginal vault as a recurrence. Recurrent prolapse was defined in our study as prolapse of the apical compartment stage 2 or higher (evaluated by pelvic organ prolapse quantification system) 


\begin{tabular}{|c|c|c|c|}
\hline Characteristics & $\begin{array}{l}\text { Sacrospinous } \\
\text { hysteropexy } \\
(n=103)\end{array}$ & $\begin{array}{l}\text { Vaginal } \\
\text { hysterectomy } \\
(n=105)\end{array}$ & Difference $(95 \% \mathrm{CI})$ \\
\hline \multicolumn{4}{|l|}{ Intraoperative period } \\
\hline Mean (SD) operating time (min) & $59(13)$ & $72(21)$ & $-13.5(-18.5$ to -8.6$)$ \\
\hline Mean (SD) estimated blood loss (mL) & $202(74)$ & $209(112)$ & $-6.5(-32.8$ to 20.0$)$ \\
\hline \multicolumn{4}{|l|}{ Complications: } \\
\hline Related to antibiotic use & $1(1)$ & $0(0)$ & $1.0(-2.2$ to 4.2$)$ \\
\hline Related to surgery & $0(0)$ & $1(1)^{\star}$ & $-1.0(-4.2$ to 2.2$)$ \\
\hline \multicolumn{4}{|l|}{ Concomitant surgery: } \\
\hline Anti-incontinence & $4(4)$ & $4(4)$ & $0.1(-5.7$ to 5.8$)$ \\
\hline Anterior colporrhaphy & $100(97)$ & $104(99)$ & $-1.9(-6.5$ to 2.6$)$ \\
\hline Posterior colporrhaphy & $30(29)$ & $52(50)$ & $-20(-33.0$ to -7.0$)$ \\
\hline Anterior and posterior colporrhaphy & $30(29)$ & $52(50)$ & $-20(-33.0$ to -7.0$)$ \\
\hline \multicolumn{4}{|l|}{ Surgeon: } \\
\hline Gynaecologist & $98(97)$ & $85(82)$ & $13.9(5.1$ to 22.7$)$ \\
\hline Resident & $3(3)$ & $19(18)$ & $-14.9(-23.2$ to -6.6$)$ \\
\hline \multicolumn{4}{|l|}{ Postoperative period } \\
\hline Mean (SD) length of hospital stay (days) & $3(1)$ & $3(1)$ & $-0.1(-0.4$ to 0.2$)$ \\
\hline \multicolumn{4}{|l|}{ Complications during hospital stay: } \\
\hline Death & $0(0)$ & $1(1)$ & $-1.0(-4.2$ to 2.2$)$ \\
\hline Reoperation because of bleeding & $0(0)$ & $1(1)$ & $-1.0(-4.2$ to 2.2$)$ \\
\hline Cerebrovascular accident & $1(1)$ & $0(0)$ & $1.0(-2.2$ to 4.2$)$ \\
\hline Buttock pain & $9(9)$ & $0(0)$ & $8.6(2.6$ to 14.5$)$ \\
\hline Urinary retention & $15(15)$ & $12(11)$ & $3.1(-6.2$ to 12.4$)$ \\
\hline Infection needing antibiotics & $3(3)$ & $0(0)$ & $2.9(-1.3$ to 7.0$)$ \\
\hline Endometrial carcinoma & $1(1)$ & $0(0)$ & $1.0(-2.2$ to 4.2$)$ \\
\hline
\end{tabular}

Percentages were calculated using non-missing data. All women were analysed as allocated (intention to treat). *Bowel injury during abdominal hysterectomy. anatomical recurrences of the posterior compartment occurred after hysterectomy with suspension of the uterosacral ligaments, which supports this view. The risk for recurrent prolapse of the anterior vaginal wall after sacrospinous hysteropexy is often discussed. We found no difference in occurrence of prolapse of the anterior vaginal wall. This is in line with previous studies: a retrospective study by Smilen and colleagues found that the occurrence of prolapse of the anterior vaginal wall was not altered by sacrospinous hysteropexy and the randomised study performed by Dietz and colleagues did not find a higher rate of prolapse of the anterior vaginal wall after sacrospinous hysteropexy (51\%) compared with vaginal hysterectomy (64\%) after one year. ${ }^{12} 38$

Reoperation rates for (recurrent) pelvic organ prolapse did not differ. Two women ended up undergoing hysterectomy after uterine preservation. In one woman this was because of persistent buttock pain. The overall rate of buttock pain after sacrospinous hysteropexy in our study (9\%) is in line with that of other studies and in most women the pain resolved spontaneously. ${ }^{30}$ Preoperative counselling should include information about the potential risk of buttock pain. Endometrial carcinoma was found in one woman during follow-up (1\%). A previous retrospective analysis of disease findings after prolapse surgery with hysterectomy showed premalignant or malignant abnormalities in 17 of 644 patients (2.6\%). ${ }^{39}$ In that study, two women $(0.3 \%)$ had endometrial cancer diagnosed. Because of the low incidence and the early diagnosis of endometrial cancer owing to blood loss, we believe that future risk of malignancy should not be regarded as a valid reason for removal of the uterus. opinion, this composite outcome measure is more clinically relevant than outcome in terms of objective pelvic organ prolapse quantification scores alone. Barber and colleagues reported on different definitions of success after surgery for pelvic organ prolapse in 2009. ${ }^{20}$ Treatment success varied widely depending on the definition used, but definitions that included the absence of vaginal bulge symptoms had the strongest relation with the women's assessment of overall improvement and treatment success. Furthermore, the authors concluded that the hymen is an important cutoff point for development of symptoms. As new trials probably will use these updated outcome definitions, we also analysed our data using the hymen as an anatomical threshold and also used the composite outcome measure of Barber and colleagues, making this trial in the future comparable to that of others.

Although the presence of posterior vaginal wall prolapse in both groups was similar before surgery, more repairs of the posterior vaginal wall (colporrhaphies) were performed in the vaginal hysterectomy group. The surgeons were free to decide on concomitant surgery and in general this was decided during surgery. One explanation might be that the surgeons thought that the more dorsal axis of the vagina after sacrospinous hysteropexy already protected against a recurrent prolapse of the posterior vaginal wall. Despite the higher number of posterior colporrhaphies, more

\section{Clinical implications and future research}

Uterus preservation has gained popularity among gynaecologists and patients during the past years. ${ }^{6-9}$ A recent trial among 213 women from multiple study sites throughout the United States found that $36 \%$ of the women preferred uterus preservation, $20 \%$ preferred hysterectomy, and $44 \%$ had no preference, assuming equal outcomes after both procedures. ${ }^{7}$ Another preference study among 100 women showed cacious alternative was available. ${ }^{8}$ This trial provides evidence for sacrospinous hysteropexy being such an alternative and therefore this study has important implications for clinical practice. Women who want to avoid hysterectomy and preserve their uterus can be reassured that sacrospinous hysteropexy was equally effective as vaginal hysterectomy after short term follow-up. However, longer follow-up is necessary, and also randomised controlled trials comparing other uterus preserving procedures are needed.

\section{Conclusions}

Based on analysis of 12 months' follow-up, we conclude that sacrospinous hysteropexy is non-inferior to vaginal hysterectomy with suspension of the uterosacral ligaments for recurrent prolapse of the apical that $60 \%$ would decline hysterectomy if an equally effi- 


\begin{tabular}{|c|c|c|c|c|c|}
\hline \multirow[b]{2}{*}{ Domains } & \multicolumn{2}{|l|}{ Before surgery } & \multicolumn{3}{|c|}{12 months after surgery } \\
\hline & $\begin{array}{l}\text { Sacrospinous } \\
\text { hysteropexy }(n=101)\end{array}$ & $\begin{array}{l}\text { Vaginal hysterectomy } \\
(\mathrm{n}=104)\end{array}$ & $\begin{array}{l}\text { Sacrospinous } \\
\text { hysteropexy } \\
(\mathrm{n}=97)\end{array}$ & $\begin{array}{l}\text { Vaginal } \\
\text { hysterectomy } \\
(\mathrm{n}=99)\end{array}$ & Pvalue* \\
\hline \multicolumn{6}{|l|}{ Urogenital distress inventoryt: } \\
\hline Overactive bladder & $0(0-44)$ & $22(0-33)$ & $0(0-11)$ & $0(0-11)$ & 0.34 \\
\hline Urinary incontinence & $17(0-33)$ & $17(0-33)$ & $0(0-17)$ & $0(0-17)$ & 0.11 \\
\hline Obstructive micturition & $8(0-33)$ & $17(0-33)$ & $0(0-0)$ & $0(0-0)$ & 0.71 \\
\hline Genital prolapse & $50(33-67)$ & $67(33-67)$ & $0(0-0)$ & $0(0-0)$ & 0.86 \\
\hline Pain & $17(0-33)$ & $17(0-33)$ & $0(0-0)$ & $0(0-0)$ & 0.86 \\
\hline \multicolumn{6}{|l|}{ Defecatory distress inventoryt: } \\
\hline Obstipation & $0(0-17)$ & $0(0-17)$ & $0(0-0)$ & $0(0-0)$ & 0.65 \\
\hline Obstructive defecation & $0(0-17)$ & $0(0-10)$ & $0(0-8)$ & $0(0-8)$ & 0.85 \\
\hline Pain & $0(0-0)$ & $0(0-0)$ & $0(0-0)$ & $0(0-0)$ & 0.42 \\
\hline Incontinence & $0(0-0)$ & $0(0-0)$ & $0(0-0)$ & $0(0-0)$ & 0.38 \\
\hline Flatus & $33(0-33)$ & $33(0-33)$ & $0(0-33)$ & $33(0-33)$ & 0.20 \\
\hline \multicolumn{6}{|c|}{ Incontinence impact questionnaireł: } \\
\hline Mobility & $11(0-33)$ & $11(0-22)$ & $0(0-11)$ & $0(0-11)$ & 0.50 \\
\hline Physical & $0(0-33)$ & $0(0-33)$ & $0(0-0)$ & $0(0-0)$ & 0.81 \\
\hline Social & $11(0-22)$ & $0(0-11)$ & $0(0-0)$ & $0(0-0)$ & 0.99 \\
\hline Embarrassment & $0(0-17)$ & $0(0-17)$ & $0(0-0)$ & $0(0-0)$ & 0.12 \\
\hline Emotion & $0(0-33)$ & $0(0-22)$ & $0(0-0)$ & $0(0-0)$ & 0.56 \\
\hline \multicolumn{6}{|l|}{ Short form-36§: } \\
\hline Physical functioning & $80(55-90)$ & $80(65-90)$ & $90(75-100)$ & $85(70-95)$ & 0.27 \\
\hline Social functioning & $94(75-100)$ & $88(75-100)$ & $100(88-100)$ & $100(75-100)$ & 0.20 \\
\hline Role limitations physical & $75(6-100)$ & $100(50-100)$ & $100(100-100)$ & $100(75-100)$ & 0.89 \\
\hline Role limitations emotional & $100(75-100)$ & $100(100-100)$ & $100(100-100)$ & $100(100-100)$ & 0.78 \\
\hline Mental health & $84(72-92)$ & $84(72-88)$ & $84(76-92)$ & $84(72-92)$ & 0.57 \\
\hline Vitality & $70(50-80)$ & $70(55-80)$ & $75(55-80)$ & $75(65-80)$ & 0.39 \\
\hline Bodily pain & $78(59-100)$ & $80(67-100)$ & $100(67-100)$ & $100(78-100)$ & 0.92 \\
\hline General health perception & $75(55-85)$ & $75(61-85)$ & $75(60-90)$ & $75(60-90)$ & 0.72 \\
\hline Health change & $50(25-50)$ & $50(50-50)$ & $75(50-100)$ & $75(50-100)$ & 0.52 \\
\hline \multicolumn{6}{|c|}{$\begin{array}{l}\text { All patients were analysed as allocated. } \\
\text { *P value for exploratory purposes: Mann-Whitney } U \text { test of sacrospinous hysteropexy versus vaginal hysterectomy. } \\
+0=\text { no symptoms or not bothersome to } 100=\text { most bothersome symptoms. } \\
\neq 0=\text { best quality of life to } 100=\text { worst quality of life. }\end{array}$} \\
\hline
\end{tabular}

\begin{tabular}{|c|c|c|c|c|c|}
\hline \multirow[b]{2}{*}{ Time point of assessment } & \multicolumn{2}{|c|}{ Sacrospinous hysteropexy } & \multicolumn{2}{|c|}{ Vaginal hysterectomy } & \multirow[b]{2}{*}{ Pvalue* } \\
\hline & No of women & Mean (SD) score & No of women & Mean (SD) score & \\
\hline \multicolumn{6}{|l|}{ Recovery index-10t: } \\
\hline Week 1 & 99 & $32(7)$ & 99 & $33(6)$ & 0.66 \\
\hline Week 2 & 100 & $34(7)$ & 99 & $34(7)$ & 0.58 \\
\hline Week 4 & 98 & $36(7)$ & 98 & $36(6)$ & 0.82 \\
\hline Week 6 & 98 & $38(8)$ & 99 & $38(9)$ & 0.87 \\
\hline \multicolumn{6}{|l|}{ PISQ-12キ: } \\
\hline Total score at baseline & 56 & $33(6)$ & 64 & $35(5)$ & 0.05 \\
\hline Total score at 12 months & 49 & $37(5) \S$ & 56 & $37(4) \S$ & 0.62 \\
\hline \multicolumn{6}{|c|}{$\begin{array}{l}\text { PISQ-12=pelvic organ prolapse/urinary incontinence sexual questionnaire. } \\
\text { *P value for exploratory purposes: independent samples t test of sacrospinous hysteropexy versus vaginal hysterectomy. } \\
+10 \text { item questionnaire measuring postoperative recovery on } 5 \text { point Likert scale. Summary scale score ranges from } 10 \text { to } 50 \text {, where } 50 \text { indicates perfect } \\
\text { recovery. } \\
\text { fTotal scores range from } 0 \text {, which represents poorest sexual function, to } 48 \text { best sexual function. } \\
\text { §Not shown: paired sample test baseline score and follow-up score (sacrospinous hysteropexy } P<0.002 \text { and vaginal hysterectomy } P<0.002 \text { ). }\end{array}$} \\
\hline
\end{tabular}

compartment with bothersome bulge symptoms or repeat surgery for recurrent apical prolapse. Overall anatomical outcome, functional outcome, hospital stay, complications, postoperative recovery, and sexual functioning did not differ.
We thank the staff at the participating centres for their assistance with study enrolment. We also thank N van Rijn for help with data collection and administrative support.

Contributors: RJD, JdB, MEV, and HWFvE conceived and designed the study. RJD, JdB, JS, and HWFVE acquired the data. RJD, Jl, and KBK carried out the statistical analysis. RJD and HWFvE obtained funding 
and supervised the study and are the guarantors. RJD, JdB, and HWFvE provided administrative, technical, or material support. All authors analysed and interpreted the data, drafted the manuscript, critically revised the manuscript for important intellectual content, had full access to all of the data in the study, and take responsibility for the integrity of the data and the accuracy of the data analysis.

Funding: This study received an unrestricted grant from the Isala research foundation. The funding source had no role in the design and conduct of the study; collection, management, analysis, or interpretation of the data; or preparation, review, or approval of the manuscript.

Competing interests: All authors have completed the ICMJE uniform disclosure form at www.icmje.org/coi disclosure.pdf and declare: IdB was a scientific medical advisor for Astellas and received consultancy fees, and has written an e-learning on incontinence for general practitioners. MEV is an advisory committee member for Astellas and receives consultancy fees. HWFvE receives training honorariums from Johnson \& Johnson, AMS, and Bard Medical.

Ethical approval: This study was approved by the medical ethical committee of the Isala hospital (MEC 09-625) and the local ethical committees of the participating centres, in accordance with the Declaration of Helsinki.

Data sharing: No additional data available.

Transparency: The corresponding author (RJD) affirms that the manuscript is an honest, accurate, and transparent account of the study being reported, no important aspects of the study have been omitted, and any discrepancies from the study as planned have been explained.

This is an Open Access article distributed in accordance with the Creative Commons Attribution Non Commercial (CC BY-NC 4.0) license, which permits others to distribute, remix, adapt, build upon this work non-commercially, and license their derivative works on different terms, provided the original work is properly cited and the use is non-commercial. See: http://creativecommons.org/licenses/ by-nc/4.0/.

1 Slieker-ten Hove MC, Pool-Goudzwaard AL, Eijkemans MJ, Steegers-Theunissen RP, Burger CW, Vierhout ME. The prevalence of pelvic organ prolapse symptoms and signs and their relation with bladder and bowel disorders in a general female population. Int Urogynecol J Pelvic Floor Dysfunct 2009;20:1037-45.

2 Olsen AL, Smith VI, Bergstrom JO, Colling JC, Clark AL. Epidemiology of surgically managed pelvic organ prolapse and urinary incontinence. Obstet Gynecol 1997;89:501-6.

3 De Boer TA, Slieker-ten Hove MC, Burger CW, Kluivers KB, Vierhout ME. The prevalence and factors associated with previous surgery for pelvic organ prolapse and or urinary incontinence in a cross sectional study in the Netherlands. Eur J Obstet Gynecol Reprod Biol 2011;158:343-9.

4 Jha S, Moran P. The UK national prolapse survey: 5 years on. Int Urogynecol J 2011;22:517-28

5 Vanspauwen R, Seman E, Dwyer P. Survey of current management of prolapse in Australia and New Zealand. Aust N Z J Obstet Gynaecol 2010;50:262-7.

6 Detollenaere RJ, den Boon J, Kluivers KB, Vierhout ME, van Eijndhoven HW. Surgical management of pelvic organ prolapse and uterine descent in the Netherlands. Int Urogynecol / 2013:24:781-8.

7 Korbly NB, Kassis NC, Good MM, et al. Patient preferences for uterine preservation and hysterectomy in women with pelvic organ prolapse. Am J Obstet Gynecol 2013;209:470.e1-6.

8 Frick AC, Barber MD, Paraiso MF, Ridgeway B, Jelovsek JE, Walters MD. Attitudes toward hysterectomy in women undergoing evaluation for uterovaginal prolapse. Female Pelvic Med Reconstr Surg 2013;19:103-9.

9 Wu MP, Long CY, Huang KH, Chu CC, Liang CC, Tang CH. Changing trends of surgical approaches for uterine prolapse: an 11-year population-based nationwide descriptive study. Int Urogynecol J 2012;23:865-72

10 Maher CF, Cary MP, Slack MC, Murray CJ, Milligan M, Schluter P. Uterine preservation or hysterectomy at sacrospinous colpopexy for uterovaginal prolapse? Int Urogynecol J Pelvic Floor Dysfunct 2001;12:381-4

11 Brummen HJ, van de Pol G, Aalders CIM, Heintz APM, van der Vaart. Sacrospinous hysteropexy compared to vaginal hysterectomy as primary surgical treatment for a descensus uteri: effect on urinary symptoms. Int Urogynecol J Pelvic Floor Dysfunct 2003;14:350-5.

12 Hefni MA, El-Toukhy TA, BhaumikJ, Katsimanis E. Sacrospinous cervicocolpopexy with uterine conservation for uterovaginal prolapse in elderly women: an evolving concept. Am J Obstet Gynecol 2003;188:645-50
13 Dällenbach P, Kaelin-Gambirasio I, Jacob S, Dubuisson JB, Boulvain M Incidence rate and risk factors for vaginal vault prolapse repair after hysterectomy. Int Urogynecol J Pelvic Floor Dysfunct 2008;19:1623-9.

14 Kantartzis KL, Turner LC, Shepherd JP, Wang L, Winger DG, Lowder JL. Apical support at the time of hysterectomy for uterovaginal prolapse. Int Urogynecol / 2015;26:207-12.

15 Bulletins-Gynecology ACoP. ACOG practice bulletin No 85: pelvic organ prolapse. Obstet Gynecol 2007;110:717-29.

16 Dietz V, van der Vaart CH, van der Graaf Y, Heintz P, Schraffordt Koops SE. One-year follow-up after sacrospinous hysteropexy and vaginal hysterectomy for uterine descent: a randomized study. Int Urogynecol J Pelvic Floor Dysfunct 2010;2:209-16.

17 Jeng CI, Yang YC, Tzeng CR, Shen J, Wang LR. Sexual functioning after vaginal hysterectomy or transvaginal sacrospinous hysteropexy for uterine prolapse: a comparison. J Reprod Med 2005;50:669-74.

18 Maher C, Feiner B, Baessler K, Schmid C. Surgical management of pelvic organ prolapse in women. Cochrane Database Syst Rev 2013;4:CD004014

19 Detollenaere RJ, den Boon J, Stekelenburg J, et al. Treatment of uterine prolapse stage 2 or higher: a randomized multicenter trial comparing sacrospinous fixation with vaginal hysterectomy (SAVE U trial). BMC Womens Health 2011;11:4.

20 Barber MD, Brubaker L, Nygaard I, et al. Defining success after surgery for pelvic organ prolapse. Obstet Gynecol 2009;114:600-9.

21 Cruikshank SH. Preventing posthysterectomy vaginal vault prolapse and enterocele during vaginal hysterectomy. Am J Obstet Gynecol 1987:156:1433-40.

22 Bump RC, Mattiasson A, Bo K, et al. The standardization of terminology of female pelvic organ prolapse and pelvic floor dysfunction. Am J Obstet Gynecol 1996;175:10-7.

23 Ware JE, Kosinski M, Keller SD. SF-36 physical and mental component summary measures-a users' manual. 1994. New England Medical Center, The Health Institute.

24 Van der Vaart CH, de Leeuw JR, Roovers IP, Heintz AP. Measuring health-related quality of life in women with urogenital dysfunction: the urogenital distress inventory and incontinence impact questionnaire revisited. Neurourol Urodyn 2003;22:97-104

25 Roovers JP, van der Bom JG, van der Vaart CH, Heintz AP. Prediction of findings at defecography in patients with genital prolapse. Br J Obstet Gynaecol 2005;112:1547-53.

26 Lamers LM, Stalmeier PF, McDonnell J, Krabbe PF, van Busschbach J]. Measuring the quality of life in economic evaluations: the Dutch EQ-5D tariff. Ned Tijdschr Geneeskd 2005;149:1574-8.

27 Schweitzer KJ, de Jong M, Milani AL. Prolaps en seks: hoe meten we de relatie? Ned Tijdschr Obst Gyn 2008;121:79-82.

28 Kluivers KB, Hendriks JC, Mol BW, et al. Clinimetric properties of 3 instruments measuring postoperative recovery in a gynecologic surgical population. Surgery 2008:144:12-21

29 Dietz V, Schraffordt Koops SE, van der Vaart CH. Vaginal surgery for uterine descent; which options do we have? A review of the literature. Int Urogynecol J Pelvic Floor Dysfunct 2009;20:349-56.

30 Dietz V, Schraffordt Koops S, van der GraafY, Heintz P, van der Vaart C. Sacrospinous hysteropexy and vaginal hysterectomy for uterine descent: a randomized study. Int Urogynecol / 2008;19:S1-166.

31 Agresti A, Coull B. Approximate is better than 'exact' for interval estimation of binomial proportions. Am Stat 1998;52:119-26.

32 Bland JM, Altman DG. Multiple significance tests: the Bonferroni method. BM/ 1995;310:170

33 Lykke R, Blaakær J, Ottesen B, Gimbel H. Pelvic organ prolapse (POP) surgery among Danish women hysterectomized for benign conditions: age at hysterectomy, age at subsequent POP operation, and risk of POP after hysterectomy. Int Urogynecol / 2015;26:527-32.

34 Altman D, Falconer C, Cnattinguis S, Granath F. Pelvic organ prolapse surgery following hysterectomy on benign indications. Am J Obstet Gynecol 2008;198:572.e1-6.

35 McCall ML. Posterior culdoplasty; surgical correction. Obstet Gynecol 1957:10:595.

36 Barber MD, Brubaker L, Burgio KL, et al. Comparison of 2 transvaginal surgical approaches and perioperative behavioral therapy for apical vaginal prolapse: the OPTIMAL randomized trial. JAMA 2014;311:1023-34

37 Gutman R, Maher C. Uterine-preserving POP surgery. Int Urogynecol) 2013;24:1803-13.

38 Smilen SW, Saine J, Wallach SJ, Porges RF. The risk of cystocele after sacrospinous ligament fixation. Am J Obstet Gynecol 1998;179:1465-71

39 Renganathan A, Edwards R, Duckett JR. Uterus conserving prolapse surgery - what is the chance of missing a malignancy? Int Urogynecol J 2010;21:819-21.

(c) BMJ Publishing Group Ltd 2015 